\title{
Significance of crystallochemical factors in chemical reactions into the structure of solids
}

\author{
Leszek Stoch $\cdot$ Paweł Stoch
}

Bretsznajder Special Chapter

(C) The Author(s) 2012. This article is published with open access at Springerlink.com

\begin{abstract}
The chemical reaction of the formation of compounds within the structure of solid as a reaction medium-internal reactions-is the subject of the article. The mechanism of these reactions on the example silicate mineral structure and nanocrystallization of oxide glasses is considered. Local atoms interaction analysis based on the electronegativity of and ionicity of the chemical bonds values helps to understand this mechanism and predict course of intrastructural thermal processes.
\end{abstract}

Keywords Solid state reactions - Electronegativity · Glass nanocrystallization

\section{Introduction}

In the inorganic materials and minerals one can observe processes occurring within the bulk of the crystal grain. The solid products of these processes are formed inside the structural framework of the parent substance (precursor) by rearrangement of the elements of this structure. Some of them proceed without any visible change of the outer form of the parent crystals (pseudomorphism of minerals). The term internal reactions of solids was proposed for this group of processes [1,2].They are the chemical reaction of the formation of compounds within the structure of solid as a reaction medium. Chemical components of the parent

\section{Stoch $(\bowtie)$}

Krakow Division, Institute of Ceramic and Building Materials in Warsaw, Warsaw, Poland

e-mail: stoch_1@poczta.onet.pl

P. Stoch

Faculty of Materials Science and Ceramics, AGH Technical

University of Science and Technology, Krakow, Poland structure are reaction substrates and solid product of the reaction is formed within the precursor grain. These reactions seemingly do not obey the fundamental rules of classic chemistry, it is mass action rule; tendency of the system to obtain state of minimum of energy (minimum of $\mathrm{dG}$ or $\mathrm{dF}$ of the process); and crystallization of products according to nucleation-crystal growth mechanism.

Internal reactions take place through the displacement of ions inside the parent structure. Diffusional transport of particular components in solid is slow, differentiated by parent structure and its rate is changing with temperature increase. As a consequence only these components which at the given temperature posses the appropriate freedom of displacement and mobility may participate in internal reactions. The result is distinctly multi-stage course of many internal reactions and step by step, through metastable phases formation and their evolution are the mode of establishing equilibrium state. Wellknown empiric Oswald's rule of steps expresses this.

Composition of products of particular stages of the internal reactions can be dependent on the local concentration of elements mobile enough to interact and not on chemical composition of solid in a bulk. There are a number of substances undergoing thermal reactions of internal character is considerable, particularly amongst those whose structure has a polymeric character, such as silicates, borates phosphates and glasses. In geology, minerals transformation by diffusional-ionic metasomatism belongs to internal reactions. Factors governing the internal reactions direction and rate are: 1 . local chemical affinity of elements components of the primary structure, 2. progress of the primary structure destruction and 3 . reactants mobility.

For better understanding of internal reaction mechanism and to predict their direction, the crystallochemical factors characterizing chemical bonds and atomic interactions were applied. 
The electronegativity and ionicity of chemical bonds values are used as the measure of the atomic interactions. Usefulness of the crystallochemical analysis of the local atoms interactions for understanding internal reactions mechanism and metastable phases formation has been proved in the studies of thermal decomposition of minerals and early stages of the crystallization of glass and nanocrystalline materials formation. Some examples of them are presented below.

\section{Local atoms interactions analysis}

Electronegativity is widely accepted indicator of character of the chemical bonds of elements and as the same their chemical properties. The concept of the electronegativity of chemical elements was introduced by L. Pauling (1932). According to his definition, electronegativity $\left(x_{\mathrm{p}}\right)$ describes the ability of atoms to attract electrons in chemical bonds. Values $x_{\mathrm{p}}$ were calculated from thermal dissociation energies (thermochemical electronegativity), and electronegativity scale of elements was arbitrary established giving for hydrogen electronegativity value $x_{\mathrm{p}}=0$. The most modern concepts of electronegativity are linked with the ionization energies of free atoms and postulate conservation of the individuality of atoms in the bonds.

In our consideration, we use the electronegativity concept formulated by Görlich as newest one and describing precisely the properties of electronic shape of atom or ion. According to his definition, electronegativity of the atomic core or cation $\left(x_{\mathrm{G}}\right)$ is equivalent to the respective effective nuclear charges $Z_{\text {effv }}$ in $\mathrm{eV}$ units. Its value is calculated from ionisation energy: $x_{\mathrm{G}}=Z_{\mathrm{effv}}=\sqrt{I_{\mathrm{V}}^{\prime}}$, where $I_{\mathrm{v}}^{\prime}$ is the ionisation energy in the Rydberg units, needed to remove the $\mathrm{v}$ valency electrons from an atom. Atomic core is atomic nucleus with those electrons which remained after all valence electrons were removed from atom by ionization. The atomic cores are representative for chemical elements so $Z_{\mathrm{effv}}$ values represent electronegativity of chemical elements. Chemical properties of element are decisively dependent on its valency state, and in this case, effective nuclear charge for cations $Z_{\text {effz }}$ is used, where $\mathrm{z}$ is oxidation state of cation, it is the number of valency electrons taking part in chemical bond between atoms. The close relation exists between electronegativity scale by Görlich and Pauling's scale [3, 4]. The electronegativity $Z_{\text {effv }}$ and atomic number of elements in periodic system $Z$ corresponds together and can be considered as two fundamental concepts functioning in chemistry [5].

The relative difference of the nuclear charge of the two bonding atoms constitutes the factor determining the ionicity of the heteropolar bond. The ionicity $i_{\mathrm{G}}$ or asymmetry of the chemical bonds between different atomic cores or ions, as cation-oxygen bond is defined by the relation $i_{\mathrm{G}}=1-Z_{\mathrm{eff} 1} / Z_{\text {eff2 }}$, where $Z_{\text {eff } 1}<Z_{\text {eff2 }}$. The intrinsic affinity between two different atoms forming a heteronuclear compound is directly connected with the value of ionicity of a chemical bond. In the presented studies, the ionicity value of bonds of the components atoms with oxygen $\left(i_{\mathrm{G}}\right)$ has been applied as a parameter characterising the strength of the bonds. It increases with decreasing ionicity. Another parameter is the 'localisation of the bonding electron', $L=\bar{Z}_{\text {eff }}=\sqrt{Z_{\text {eff } 1} \cdot Z_{\text {eff } 2}}[4,5]$. Its value increases with the covalence of the bonds with oxygen. This parameter has been accepted by us as a measure of rigidity of the bonds.

The increase in the proportion of the non-directional ionic bond makes the bond more flexible, increase of the covalence makes the bond more and more directed; the structure is more rigid but simultaneously increases its strength. Structure of oxides, especially polymeric like silicates, phosphates and borates, can be considered as a net formed by cations joined by oxygen ions-oxide bridges. In this structure, oxygen behaves as Lewis bases transferring part of their negative charge to the cations as acidic components of the structure. The acid-base interaction along the oxygen bridge determines the mutual chemical affinity of joined components. The ionicity $\left(x_{\mathrm{G}}\right)$ of $\mathrm{Me}-\mathrm{O}$ bond can be used as a measure of the basicity of cation, higher ionic of the bond more basic cation. Difference in ionicity of cations forming oxygen bridge $\mathrm{Me}-\mathrm{O}-\mathrm{Me}, \Delta i_{\mathrm{G}}$ was taken as the measure of local chemical affinity of the joining cations: $A=\Delta i_{\mathrm{G}}=i_{\mathrm{Gb}}-i_{\mathrm{Ga}}$ (where $i_{\mathrm{Gb}}$ is the ionicity of basic and $i_{\mathrm{Ga}}$ is the ionicity of acidic component) at the same time $\Delta i_{\mathrm{G}}$ is estimated measure of the strength of the bridge. Difference in parameter $L$ value of $\mathrm{Me}-\mathrm{O}$ bonds of high degree of covalency forming the bridge was taken as indicator of internal strains caused by their rigidity. The $\mathrm{Si}-\mathrm{O}-\mathrm{P}$ bridge is an example.

\section{Internal thermal reactions: examples}

Crystal structure rebuilding process

Kaolinite thermal reactions are the best example of the crystal structure rebuilding. Kaolinite, the layer structure aluminium silicate $2 \mathrm{Al}_{2} \mathrm{O}_{3} \cdot 4 \mathrm{SiO}_{2} \cdot 4 \mathrm{H}_{2} \mathrm{O}$, consists of the continuous sheet, formed from ${ }^{\mathrm{IV}}\left[\mathrm{SiO}_{4}\right]$ tetrahedra and octahedral $^{\mathrm{VI}}(\mathrm{Al}, \mathrm{O}, \mathrm{OH})$ sheet joined together with $\mathrm{Si}-\mathrm{O}-$ Al bridges into the common layer. Between the adjacent layers hydrogen bonds exist. The thermal reactions of kaolinite consist of dehydroxylation stage, and the anhydrous structure rebuilding stage. At the dehydroxylation, 
the adjacent $\mathrm{OH}$ groups form $\mathrm{H}_{2} \mathrm{O}$ molecules. They escape through interlayer spaces in the kaolinite structure. When heating is fast enough, the dehydroxylation is explosive and the layers are separated [6].

Dehydroxylated kaolinite, named metakaolinite has X-ray amorphous structure. It consists of the preserved silica-oxygen tetrahedral sheet but octahedral sheet is destroyed and transformed into groups of Al-O tetrahedra. The outer form of kaolinite plates is preserved (Fig. 1a, b).

At $970{ }^{\circ} \mathrm{C}$ (sharp DTA exothermic peak), rebuilding of the metakaolinite internal structure takes place ( $\mathrm{Al}, \mathrm{Si}$ ), spinel like phase in amorphous silica $\left(\mathrm{SiO}_{2}\right)$ matrix is formed. Structure of the spinel is rebuilt and next quantities $\mathrm{SiO}_{2}$ expelled. Finally, about $1,200{ }^{\circ} \mathrm{C}$ (next exothermic peak) equilibrium composition state is attained and mullite of the perfect $3 \mathrm{Al}_{2} \mathrm{O}_{3} \cdot 2 \mathrm{SiO}_{2}$ composition and structure and cristobalite $\left(\mathrm{SiO}_{2}\right)$ crystallize. The process is described in [7]. Internal rebuilding of the metakaolinite $\left(2 \mathrm{Al}_{2} \mathrm{O}_{3}\right.$. $\left.4 \mathrm{SiO}_{2}\right)$ structure to the mullite $\left(3 \mathrm{Al}_{2} \mathrm{O}_{3} \cdot 2 \mathrm{SiO}_{2}\right)$ goes as follows:
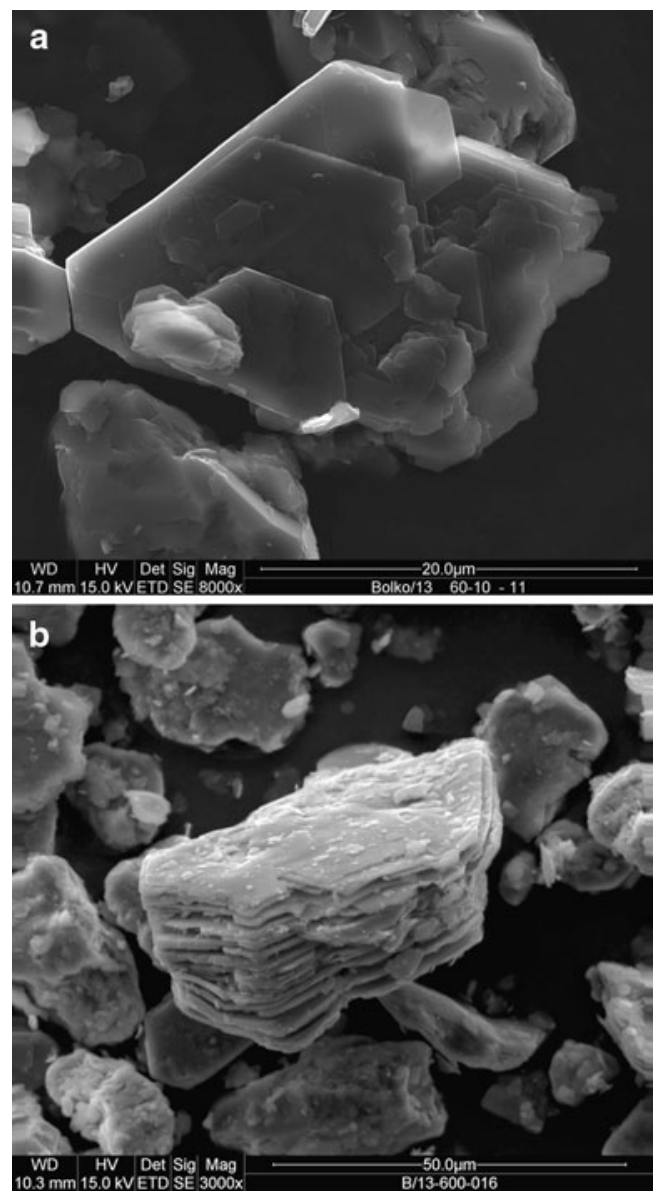

Fig. 1 Crystals of the kaolinite in natural state (a), and after dehydroxylation at $650{ }^{\circ} \mathrm{C}(\mathbf{b})$

$$
\begin{aligned}
2 \mathrm{Al}_{2} \mathrm{O}_{3} \cdot 4 \mathrm{SiO}_{2} & \rightarrow 2 \mathrm{Al}_{2} \mathrm{O}_{3} \cdot 3 \mathrm{SiO}_{2}+\mathrm{SiO}_{2} \\
& \rightarrow 2\left(2 \mathrm{Al}_{2} \mathrm{O}_{3} \cdot 2 \mathrm{SiO}_{2}\right) \\
& \rightarrow 3 \mathrm{Al}_{2} \mathrm{O}_{3} \cdot 2 \mathrm{SiO}_{2}+\mathrm{SiO}_{2}
\end{aligned}
$$

Dehydroxylation and the next rearrangements take place inside the persisting kaolinite plate, which one can consider as the individual thermodynamic system.

The mechanism of these rearrangements is well understood if the ionicity of chemical bonds was taken into account. Bond $\mathrm{Si}-\mathrm{O}$ is strong covalent-ionic type with ionicity $x_{\mathrm{G}}=0.428$. It makes that silica-oxygen sheet is very stable and stabilizes all the structure. Al-O bond is more ionic, $x=0.549$ and at the same weaker. The octahedral sheet patches of Al-O tetrahedra appear. They are linked with the silicate sheet by $\mathrm{Si}-\mathrm{O}-\mathrm{Al}$ bridges.

Above the $970{ }^{\circ} \mathrm{C}$ (DTA sharp exothermic peak), thermal vibrations break the $\mathrm{Si}-\mathrm{O}-\mathrm{Al}$ bridges, liberated silica forms $\mathrm{SiO}_{2}$ glass and cristobalite, but aluminium-silicate domains enrich in $\mathrm{Al}$ up to mullite composition are formed, and it crystallizes $\left(1,200{ }^{\circ} \mathrm{C}\right.$ peak $)$.

\section{Nanocrystallization of glass}

Nanocrystalline of glass-ceramics is produced by proper heat treating of a solidified glass samples. Usually, they are polycomponent glasses containing admixture of the component acting as nucleator. It induces crystallization of glass and crystals of a nanosize appear at low temperature, close to glass transformation $T_{\mathrm{g}}$. With further heating, temperature increase and glass viscosity diminishing the growth of the precipitated nanocrystals takes place (recrystallization) and usually a new crystal phases are formed. Their composition depends on the concentration of main components of glass.

Thus the mechanism of near $T_{\mathrm{g}}$ temperature crystallization should significantly differ from the mechanism described by the classical nucleation theory (CNT) which was formulated for the ideal liquid. Recent investigations suggest that it proceeds by successive displacement and local ordering of the groups of atoms forming middle range order clusters (structural inheritance) and is dependent on mobility of components, increasing with temperature. Metastable intermediate phases formation is characteristic for this stage of the crystallization. The crystallization of glass in the solidified form requires its heating above $T_{\mathrm{g}}$ to weaken the bonds in the glass structure and to begin to break them step by step. The breaking of the appropriate amount of the chemical bonds in solid body is necessary to make some components of the glass sufficiently mobile to enable their reorientation and reassembling, required to form the areas of ordered crystal structure.

Examples of the $T_{\mathrm{g}}$ nanocrystallization of glass and crystallization at the glass melt described in [2] are 
Table 1 Multi-stage crystallization of glass (examples)

\begin{tabular}{lll}
\hline Glass & Viscoelastic state & Liquid state \\
\hline $\mathrm{Na}_{2} \mathrm{O}(8 \mathrm{~mol} \%)-\mathrm{MgO}(17 \mathrm{~mol} \%)-\mathrm{Al}_{2} \mathrm{O}_{3}(75-x)-\mathrm{P}_{2} \mathrm{O}_{5}$ & $\mathrm{AlPO}_{4}(\mathrm{I})-\mathrm{Mg}_{3}\left(\mathrm{PO}_{4}\right)_{2}$ in glassy matrix & \\
$\quad(75-y)-\mathrm{SiO}_{2}(75-\mathrm{z})$ & & $\mathrm{AlPO}(\mathrm{II})$ in melt \\
$\mathrm{K}_{2} \mathrm{O}(10 \mathrm{wt} \%)-\mathrm{CaO}(15 \mathrm{wt} \%)-\mathrm{MgO}(10 \mathrm{wt} \%)-$ & $\mathrm{Ca}_{3}\left(\mathrm{PO}_{4}\right)-\mathrm{Mg}_{2} \mathrm{SiO}_{4}$ in glassy matrix & \\
$\quad \mathrm{Al}_{2} \mathrm{O}_{3}(10 \mathrm{wt} \%)-\mathrm{P}_{2} \mathrm{O}_{5}(15 \mathrm{wt} \%)-\mathrm{SiO}_{2}(40 \mathrm{wt} \%)$ & & $\mathrm{KAlSiO}_{4}$ in melt \\
$\mathrm{LaF}_{3}(3.0 \mathrm{wt} \%)-\mathrm{Na}_{2} \mathrm{O}(15.8 \mathrm{wt} \%)-\mathrm{Al}_{2} \mathrm{O}_{3}$ & $\mathrm{LaF}_{3}-\mathrm{NaLa}_{9}\left(\mathrm{SiO}_{6}\right) \mathrm{O}_{2}$ in glassy matrix & \\
$(24.8 \mathrm{wt} \%)-\mathrm{SiO}_{2}(56.4 \mathrm{wt} \%)$ & & $\mathrm{NaAlSiO}_{4}$ in melt \\
\hline
\end{tabular}

presented in Table 1. In the glass network built of the tetrahedra $\left[\mathrm{SiO}_{4}\right]$ and $\left[\mathrm{PO}_{4}\right]$ bonds differing considerably in their ionicity appear. This refers in particular to $\mathrm{Si}-\mathrm{O}$ bonds $\left(i_{\mathrm{G}}=0.428, L=2.410\right)$ and $\mathrm{P}-\mathrm{O}$ bonds $\left(i_{\mathrm{G}}=\right.$ $0.314, L=2.640)$. Such a large difference in bonds $(\Delta L)$, which are rigid, due to the high degree of covalency $(L>2.100)$ is responsible for the fact that in the oxygen bonds, joining both tetrahedra, considerable strains will appear, which make the bonds breakable. That is why glasses with a two component network $\mathrm{SiO}_{2}-\mathrm{P}_{2} \mathrm{O}_{5}$ are of low stability. The earlier study [7] revealed that introduction of aluminium to phosphate glasses eliminates the $\mathrm{P}=\mathrm{O}$ bonds and $\mathrm{Si}-\mathrm{O}-\mathrm{P}$ bridges are replaced by $\mathrm{Al}-\mathrm{O}-\mathrm{P}$ bridges. Then more stable links of $\left[\mathrm{PO}_{4}\right]$ and $\left[\mathrm{SiO}_{4}\right]$ tetrahedra by means of $\left[\mathrm{AlO}_{4}\right]$ are formed. The oxygen bridges $\mathrm{Al}-\mathrm{O}-\mathrm{Si}$ and $\mathrm{Al}-\mathrm{O}-\mathrm{P}$ formed then have their bonds less differing, than those in the $\mathrm{Si}-\mathrm{O}-\mathrm{P}$ bridge. However, a certain asymmetry of the bond is still retained, which results from the stronger interaction within the Al-O-P bridge $\left(\Delta i_{\mathrm{G}}=\right.$ $0.232)$ in comparison with Al-O-Si bridge $\left(\Delta i_{\mathrm{G}}=0.118\right)$ and the latter is easier to break. Thanks to this, the groups $\left[\mathrm{AlO}_{4}\right]\left[\mathrm{PO}_{4}\right]$ can be released from the silicate network and the crystallisation of $\mathrm{AlPO}_{4}$ takes place already near $T_{\mathrm{g}}$, when the glass is heated.

The release of phosphate domains of the network enables a simultaneous formation of crystalline calcium or calcium-magnesium phosphates. The chemical affinity of $\mathrm{CaO}$ to $\mathrm{P}_{2} \mathrm{O}_{5}$ expressed as the difference in ionicity of their bonds with oxygen $\left(\Delta i_{\mathrm{G}}=0.456\right)$ is much larger than for $\mathrm{SiO}_{2}\left(\Delta i_{\mathrm{G}}=0.279\right)$ and the bonds in oxygen bridges are correspondingly stronger. The $\mathrm{La}_{2} \mathrm{~F}_{6}$ nanocrystallization (Table 1) is described in [8]; the usefulness of the local atoms interaction analysis was confirmed in many articles, for example [8-12].

\section{Conclusions}

Local atoms interactions analysis based on the electronegativity of and ionicity of the chemical bonds values helps to understand the mechanism and predict course of intrastructural thermal processes. It is particularly useful in the study of the formation and existence of thermodynamic metastable states in the solid-state chemical reactions.

Acknowledgements The study was partly supported by the Project No. N N507 235740 of the National Science Centre of Poland.

Open Access This article is distributed under the terms of the Creative Commons Attribution License which permits any use, distribution, and reproduction in any medium, provided the original author(s) and the source are credited.

\section{References}

1. Stoch L. Internal structure rebuilding reactions of crystalline and amorphous solids. J Therm Anal Calorim. 1992;38:131-9.

2. Stoch L. Thermochemistry of solids with flexible structure. J Therm Anal Calorim. 1998;54:9-24.

3. Görlich E. The effective nuclear charges and their relation to the Pauling's electronegativity scale. Z Phys Chem. 1989;270: 384-388, 961-967.

4. Görlich E. The effective nuclear charges and the softness of the atomic cores. Z Phys Chem. 1990;271:169-74.

5. Görlich E. The effective nuclear charges and the electronegativity. Krakow: Polish Academy of Art and Science; 1997.

6. Stoch L. Explosive thermal dehydroxylation of solids. J Therm Anal Calorim. 1991;37:1429-45.

7. Brindley GW, Nakhira M. The kaolinite-mullite reaction series. J Am Ceram Soc. 1959;42:311-20.

8. Sroda M, Wacławska I, Stoch L, Reben M. DTA/DTS study of nanocrystallization in oxyfluoride glass. J Therm Anal Calorim. 2004;77:193-2000.

9. Stoch L, Stoch P. Crystal structure formation in glass from view of HRTM. J Therm Anal Calorim. 2007;88:577-82.

10. Wacławska I, Szumera M. Influence of $\mathrm{MgO}(\mathrm{CaO})$ on the structure of silicate-phosphate glasses: TA and NMR study. J Therm Anal Calorim. 2006;84:185-90.

11. Wacławska I, Szumera M. Thermal behaviour of Fe-doped silicate-phosphate glasses. J Therm Anal Calorim. 2010;101:423-7.

12. Sulowska J, Wacławska I, Szumera M, Olejniczak K. Characterization of crystalline phases induced by thermal treatment in CuO-containing silicate-phosphate glasses. J Therm Anal Calorim. 2011. doi:10.1007/s10973-011-1988-5. 